\title{
Unique solution for a fourth-order boundary value problem
}

\author{
Youzheng Ding \\ Department of Mathematics, \\ Shandong Jianzhu University, \\ Jinan, 250101, China \\ E-mail: dingyouzheng@139.com
}

\author{
Jiafa Xu \\ School of Mathematics, \\ Shandong University, \\ Jinan, 250100, China \\ E-mail: xujiafa292@sina.com
}

\begin{abstract}
This paper mainly concerns the uniqueness of solutions for a fourth order boundary value problem. By virtue of Browder theorem, the main result is obtained when the nonlinearity term $f$ satisfies the Lipschitz condition. The result is new and complement of some previously known results.
\end{abstract}

Keywords-boundary value problem; solution; uniqueness; Browder theorem

\section{INTRODUCTION}

In this paper, we shall study the unique solution for the fourth order boundary value problem

$$
\left\{\begin{array}{l}
u^{(4)}(t)+\eta u "(t)-\xi u(t)=f(t, u(t)), 0<t<1, \\
u(0)=u(1)=u "(0)=u "(1)=0
\end{array}\right.
$$

Where $f:[0,1] \times R \rightarrow R$ is continuous, $\xi, \eta \in R$ are parameters, which satisfy the conditions:

$$
\frac{\xi}{\pi^{4}}+\frac{\eta}{\pi^{2}}<1, \xi \geq-\frac{\eta^{2}}{4}, \eta<2 \pi^{2} .
$$

Recent years, there have been many papers to study the existence of solutions for some fourth order boundary value problems, see [1-5]. In [1, 2], for the problems of type (1), the authors all obtained the existence results of positive solutions when $f$ is either superlinear or sublinear in $u$ by employing the cone expansion or compression fixed point theorem. In [3-5], the authors applied critical point theory to consider the existence of solutions for a class of fourth order boundary value problems and obtained some excellent results. In this paper, by virtue of Browder theorem, we shall discuss the unique solution for the problem (1). It is interested that we only need the nonlinearity $f$ to satisfy the Lipschitz condition, it is a natural condition and there are a large number of continuous functions satisfy the condition. As is known, $C[0,1]$ is the Banach space with the norm $\|\cdot\|=\max _{t \in[0,1]}|x(t)|$ and $L^{2}[0,1]$ is a real Hilbert space with the inner product

$$
(u, v)=\int_{0}^{1} u(t) v(t) d t, \forall u, v \in L^{2}[0,1]
$$

Let

$$
\mu_{1}, \mu_{2}=\frac{-\eta \pm \sqrt{\eta^{2}+4 \xi}}{2}, w_{i}=\sqrt{\left|\mu_{i}\right|}, i=1,2,
$$

and

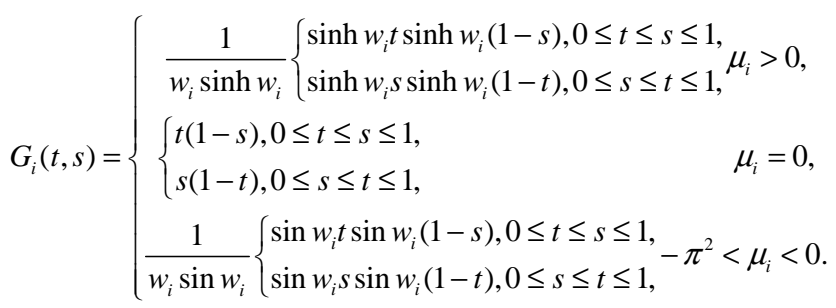

Then the problem (1) has a solution in $C^{4}[0,1]$ if and only if the following equation

$u(t)=\int_{0}^{1} \int_{0}^{1} G_{1}(t, s) G_{2}(s, \tau) f(\tau, u(\tau)) d \tau d s, t \in[0,1],(2)$

has a solution in $C[0,1]$.

From the expression of $G_{i}(i=1,2)$, we see $G_{i}(t, s)>0$, and $G_{i}(t, s)=G_{i}(s, t)$ for all $t, s \in(0,1)$. Let

$$
G(t, s)=\int_{0}^{1} G_{1}(t, \tau) G_{2}(\tau, s) d \tau, \forall t, s \in[0,1] .
$$

Clearly, from the symmetric property of $G_{1}, G_{2}$, we have $G(t, s)=G(s, t)$. Therefore, (2) is equivalent to the following integral equation

$$
u(t)=\int_{0}^{1} G(t, s) f(s, u(s)) d s, t \in[0,1] .
$$

Define the operators $T, A: C[0,1] \rightarrow C[0,1]$ by

$$
\begin{aligned}
& T u(t)=\int_{0}^{1} G(t, s) u(s) d s, \\
& A u(t)=f(t, u(t)) .
\end{aligned}
$$

Then (2) is also equivalent to the operator equation $u=T A u, u \in[0,1]$. It is well known that all eigenvalues of $T$ are $\left\{\lambda_{k}\right\}_{k \in N}=\frac{1}{k^{4} \pi^{4}-\eta k^{2} \pi^{2}-\xi}$, which have the corresponding orthonormal eigenfunctions

and

$$
\left\{e_{k}\right\}_{k \in N}=\{\sqrt{2} \sin k \pi t\}_{k \in N},
$$

$$
\lambda_{1}>\lambda_{2}>\cdots>\lambda_{k}>\cdots>0, \forall k \in N .
$$

In what follows, we list the properties of $T$ (see [3-5]): 
(a) $T: L^{2}[0,1] \rightarrow C[0,1]$ is a linear completely continuous operator and also a linear completely continuous operator from $L^{2}[0,1]$ to $L^{2}[0,1]$;

(b) $T: L^{2}[0,1] \rightarrow C[0,1]$ is positive bounded linear and symmetric, so the square root operator of $T$, $T^{\frac{1}{2}}: L^{2}[0,1] \rightarrow C[0,1]$ exists and is unique, and is also bounded linear and symmetric with $\left\|T^{\frac{1}{2}}\right\|=\|T\|^{\frac{1}{2}}$;

(c) $T^{\frac{1}{2}}: L^{2}[0,1] \rightarrow C[0,1]$ is a linear completely continuous operator and also a linear completely continuous operator from $L^{2}[0,1]$ to $L^{2}[0,1]$.

Since $T$ is linear completely continuous and symmetric, the following formulas with $T$ hold:

$$
u=\sum_{k=1}^{\infty}\left(u, e_{k}\right) e_{k}, u \in L^{2}[0,1]
$$

$T u=\sum_{k=1}^{\infty} \frac{1}{k^{4} \pi^{4}-\eta k^{2} \pi^{2}-\xi}\left(u, e_{k}\right) e_{k}, u \in L^{2}[0,1]$,

$T^{\frac{1}{2}} u=\sum_{k=1}^{\infty} \sqrt{\frac{1}{k^{4} \pi^{4}-\eta k^{2} \pi^{2}-\xi}}\left(u, e_{k}\right) e_{k}, u \in L^{2}[0,1]$.

Let $\Lambda=\sqrt{2}\left(\sum_{k=1}^{\infty} \frac{1}{k^{4} \pi^{4}-\eta k^{2} \pi^{2}-\xi}\right)^{\frac{1}{2}}$, from the page

1367 of [3], we see $\left|T^{\frac{1}{2}} u\right| \leq \Lambda\|u\|$. Then we obtain

$$
\left(\int_{0}^{1}\left|T^{\frac{1}{2}} u(t)\right|^{2} d t\right)^{\frac{1}{2}} \leq\left(\int_{0}^{1} \Lambda^{2}\|u\|^{2} d t\right)^{\frac{1}{2}} \leq \Lambda\|u\| .
$$

Next, we shall define $J(u)=u-T^{\frac{1}{2}} A T^{\frac{1}{2}} u, u \in L^{2}[0,1]$. Then we easily see that the solution $u_{0}$ of (1) is equivalent to $J\left(u_{0}\right)=0$, see Lemma 2.7 of [3].

\section{MAIN RESULTS}

We first give our basic definitions and lemmas about Browder theorem.

Definition 2.1[6, P303] Let $E$ be a reflexive real Banach space. We say $L: E \rightarrow E^{*}$ is demicontinuous if $L$ maps strongly convergent sequences in $E$ to weakly convergent sequences in $E^{*}$.

Lemma 2.1[6, Theorem5.3.22] (Browder theorem) Let $E$ be a reflexive real Banach space. Suppose that $L: E \rightarrow E^{*}$ be an operator satisfying the conditions

(i) $L$ is bounded, demicontinuous; (ii) $\lim _{\|u\| \rightarrow \infty} \frac{(L(u), u)}{\|u\|}=+\infty$;

(iii) $L$ is monotone on the space $E$, i.e., for all $u, v \in E$, we have

$$
(L(u)-L(v), u-v) \geq 0 .
$$

Then the equation $L(u)=f^{*}$ has at least one solution $u \in E$ for every $f^{*} \in E^{*}$. If, moreover, the inequality (4) is strict for all $u, v \in E, u \neq v$, then the equation $L(u)=f^{*}$ has precisely one solution $u \in E$ for every $f^{*} \in E^{*}$.

Now, we list our assumptions for the nonlinear term $f$.

(H1) $f \in C([0,1] \times R, R)$ and $f(t, 0) \neq 0$.

(H2) There is a constant $c \in\left(0, \Lambda^{-2}\right)$ such that

$$
|f(t, u)-f(t, v)| \leq c|u-v|, \forall u, v \in L^{2}[0,1],
$$

uniformly in $t \in[0,1]$.

Example 2.1 Let

$$
f(t, u)=\varepsilon \Lambda^{-2} u+\delta,
$$

where $\varepsilon \in(0,1), \delta \neq 0$. Clearly, (H1) and (H2) hold.

Theorem 2.1 If (H1), (H2) hold, then the problem (1) has only a nontrivial solution.

Proof. We first define two operators $L_{1}, L_{2}: L^{2}[0,1] \rightarrow L^{2}[0,1]$ as follows:

$$
\begin{aligned}
& \left(L_{1}(u), v\right)=\int_{0}^{1} u(t) v(t) d t \\
& \left(L_{2}(u), v\right)=\left(T^{\frac{1}{2}} A T^{\frac{1}{2}} u, v\right)=\int_{0}^{1} f\left(t, T^{\frac{1}{2}} u(t)\right) T^{\frac{1}{2}} v(t) d t .
\end{aligned}
$$

Clearly, $L_{1}$ is linearly continuous, so bounded. We shall show $L_{2}$ is bounded and continuous. By (H2), we find there exists a $C_{1}>0$ such that

$$
|f(t, u)| \leq c|u|+c_{1}, \forall u \in L^{2}[0,1],
$$

uniformly in $t \in[0,1]$. Indeed, in the inequality of (H2), let $v=0$, we have by the triangle inequality $|f(t, u)|-|f(t, 0)| \leq|f(t, u)-f(t, 0)| \leq c|u|$, for all $u \in L^{2}[0,1]$. Let $c_{1}=|f(t, 0)|>0$. Then (5) holds, as required. Therefore, we have by (5) and Holder inequality, note that (3),

$$
\left|\left(L_{2}(u), v\right)\right| \leq \int_{0}^{1}\left|f\left(t, T^{\frac{1}{2}} u(t)\right)\right|\left|T^{\frac{1}{2}} v(t)\right| d t
$$




$$
\begin{aligned}
& \leq \int_{0}^{1} c\left|T^{\frac{1}{2}} u(t)\right|\left|T^{\frac{1}{2}} v(t)\right| d t+\int_{0}^{1} c_{1}\left|T^{\frac{1}{2}} v(t)\right| d t \\
& \leq c\left(\int_{0}^{1}\left|T^{\frac{1}{2}} u(t)\right|^{2} d t\right)^{\frac{1}{2}}\left(\left.\left.\int_{0}^{1}\right|^{\frac{1}{2}} v(t)\right|^{2} d t\right)^{\frac{1}{2}}+c_{1}\left(\left.\left.\int_{0}^{1}\right|^{T^{\frac{1}{2}}} v(t)\right|^{2} d t\right)^{\frac{1}{2}} \\
& \leq c \Lambda^{2}\|u\|\|v\|+c_{1} \Lambda\|v\| .
\end{aligned}
$$

Thus $L_{2}$ is bounded.

On the other hand, by (H2), we see from Holder inequality and (3)

$$
\begin{aligned}
& \left|\left(L_{2}\left(u_{1}\right)-L_{2}\left(u_{2}\right), v\right)\right| \leq \int_{0}^{1}\left|f\left(t, T^{\frac{1}{2}} u_{1}(t)\right)-f\left(t, T^{\frac{1}{2}} u_{2}(t)\right)\right|\left|T^{\frac{1}{2}} v(t)\right| d t \\
& \quad \leq c \int_{0}^{1}\left|T^{\frac{1}{2}} u_{1}(t)-T^{\frac{1}{2}} u_{2}(t) \| T^{\frac{1}{2}} v(t)\right| d t \\
& \quad \leq c \Lambda^{2}\left\|u_{1}-u_{2}\right\|\|v\| .
\end{aligned}
$$

Consequently, $L_{2}$ is continuous. So $(J(u), v)$ is bounded and continuous, so demicontinuous. By (5) again, we get

$$
\begin{aligned}
& (J(u), u)=\int_{0}^{1} u^{2}(t) d t-\int_{0}^{1} f\left(t, T^{\frac{1}{2}} u(t)\right) T^{\frac{1}{2}} u(t) d t \\
& \geq\|u\|^{2}-\int_{0}^{1}\left(c\left|T^{\frac{1}{2}} u(t)\right|+c_{1}\right)\left|T^{\frac{1}{2}} u(t)\right| d t \\
& \geq\|u\|^{2}-c \Lambda^{2}\|u\|^{2}-c_{1} \Lambda\|u\| .
\end{aligned}
$$

So, $\lim _{\|u\| \rightarrow \infty} \frac{(J(u), u)}{\|u\|}=+\infty$.
Finally, we shall prove $J$ is monotone. Indeed,

$$
\begin{aligned}
& (J(u)-J(v), u-v)=\int_{0}^{1}|u(t)-v(t)|^{2} d t \\
& -\int_{0}^{1}\left[f\left(t, T^{\frac{1}{2}} u(t)\right)-f\left(t, T^{\frac{1}{2}} v(t)\right)\right]\left(T^{\frac{1}{2}} u(t)-T^{\frac{1}{2}} v(t)\right) d t \\
& \geq\|u-v\|^{2}-c \int_{0}^{1}\left|T^{\frac{1}{2}} u(t)-T^{\frac{1}{2}} v(t)\right|^{2} d t \\
& \geq\|u-v\|^{2}-c \Lambda\|u-v\|^{2}>0, u \neq v .
\end{aligned}
$$

Hence, by Lemma 2.1, we find the problem (1) has only a solution.

\section{REFERENCES}

[1] Z. Bai, H. Wang, On positive solutions of some nonlinear fourthorder beam equations, J. Math. Anal. Appl., 270(2002), pp. 357-368.

[2] Y. Li, Positive solutions of fourth-order boundary value problems with two parameters, J. Math. Anal. Appl., 281(2003), 477-484.

[3] Y. Yang, J.H. Zhang, Existence of solutions for some fourth-order boundary value problems with parameters, Nonlinear Anal., 69 (2008), pp. 1364-1375.

[4] F.Y. Li, Y.H. Li, Z.P. Liang, Existence of solutions to nonlinear Hammerstein integral equations and applications, J. Math. Anal. Appl., 323 (2006), pp. 209-227.

[5] F.Y. Li, Z.P. Liang, Q. Zhang, Existence and multiplicity of solutions of a kind of fourth-order boundary value problem, Nonlinear Anal., 62(2005),pp. 803-816.

[6] P. Drabek, J. Milota, Methods of Nonlinear Analysis, Applications to Differential Equations, Birkhauser Verlag AG Basel ` Boston ` Berlin, 2007. 